\title{
PAPR Reduction in OFDM via Separation of Complex Baseband Signal
}

\author{
Sudesh Gupta \\ PG Scholar, NIIST \\ Dept. of Electronics \& Comm. \\ NIIST, Bhopal
}

\author{
Prof. Ravi Shankar Mishra \\ Professor, NIIST \\ Dept. of Electronics \& Comm. \\ NIIST, Bhopal
}

\author{
Prof. Rajesh Nema \\ Asst. Professor, NIIST \\ Dept. of Electronics \& Comm. \\ NIIST, Bhopal
}

\begin{abstract}
OFDM system is the stronger base for the future wireless communication. The one with the lowest peak to average power ratio (PAPR) among these OFDM signal sequences are selected for transmission. One major Disadvantage of OFDM is the high peak-to-average Power ratio (PAPR). One investigated technique Selected Mapping (SLM) is PAPR reduction techniques for Orthogonal Frequency Division Multiplexing (OFDM). By analyzing separation of complex baseband signal in this paper we proposed a Modernize SLM algorithm (MSLM) scheme to reduce the PAPR by using the complex signal to separate into real \& imaginary parts and use as a individually phase sequence multiply real part as well as imaginary part of complex signal. Finally select the minimum PAPR signal which is achieved by real \& imaginary part and then combine both of the approach and find one of the lowest peak to average power ratio among these OFDM signal sequences for transmission. After applying different simulation on the MATLAB software it produces less complexity and improved performance.
\end{abstract}

\section{Keywords}

OFDM, PAPR, SLM and MSLM

\section{INTRODUCTION}

An ORTHOGONAL frequency division multiplexing (OFDM) system has been proposed as a standard for the future mobile radio communication system. OFDM signals have efficient spectral bandwidth, and the performance of the OFDM system over frequencyselective fading channels is better than that of the single carrier modulation system. One of the major drawbacks of OFDM system is that the OFDM signal can have high peak to average power ratio (PAPR). The high bit ratio frequency of PAPR brings on signal distortion in the nonlinear region of high power amplifier (HPA), and the signal distortion induces the degradation of bit error rate (BER).Various techniques [1-6] have been proposed previously for reducing the PAPR.

In the recent scenario in the field of frequency division multiplexing (OFDM) [7] has been regarded and used as one of the technologies for the communication systems.
Especially OFDM has been adopted for various wireless communication Systems [8] such as wireless local area networks (WLANs) [9], wireless metropolitan area networks (WMANs), digital audio broadcasting (DAB), and digital video broadcasting (DVB) [10]. OFDM is an attractive technique for achieving high data rate in the wireless communication systems and it is robust to the frequency selective fading channel [11].

SLM technique was first described by Bauml et al. where phase sequences are generated randomly [12] from set $\{ \pm 1, \pm \mathrm{j}\}$. Neumann phase sequences are used as phase sequence set in [13]. Zhou et. al. used monomial phase sequences [14] for SLM and found that the cubic phase sequences offer better PAPR reducing capability. In [15], Yang et. al. proposed a phase sequence where a small number of components of the phase sequences have magnitude larger than one while the rest of the components are exactly one. The rows of the hadamard matrix are selected as phase sequence set for PAPR reduction in SLM technique in [16]. In [17], Peng Chang et. al. found that the chaotic phase sequence set outperforms Walsh-Hadamard sequence and Shapiro-Rudin sequence set. Pseudo random interferometer code sequence was used to reduce PAPR in MC-CDMA in [18].Generation of phase sequence , which is one of the important aspects of SLM technique, is vary random in existing phase sequences set.

In the block diagram (figure 1)shown below, the generation of phase sequence, which is one of the important aspects of SLM technique, is very random in existing phase sequences sets. For example, in Shapiro-Rudin sequence, which is constructed recursively the initial sequence, is random. Same is the case with phase sequence set defined in, chaotic sequence [8] etc. In the proposed method, the row vectors of normalized Riemann matrix are selected as phase sequence set for PAPR reduction in SLM technique. Riemann matrix has a definite structure which will be seen in section 3 . Moreover, in all the existing SLM techniques with different phase sequences, the reduction in PAPR was 4-6.6dB compared to original OFDM (without any PAPR reduction techniques). In the proposed method, through simulations, a reduction of $8.9 \mathrm{~dB}$ was observed compared with original OFDM. 


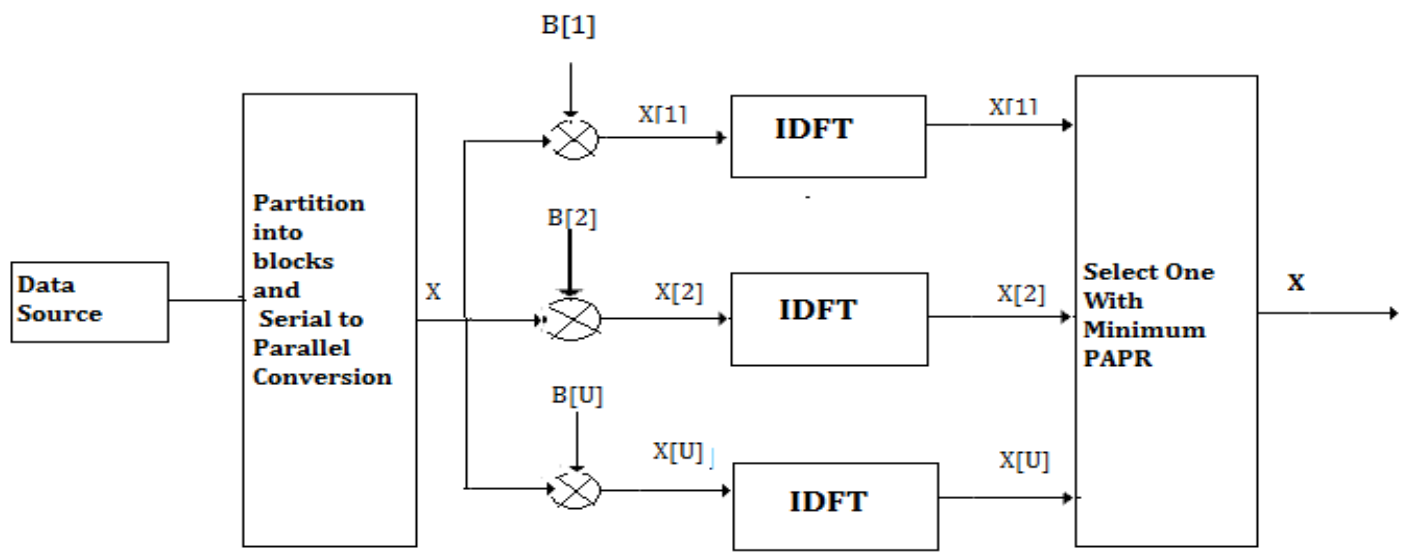

Fig 1. Block Diagram Of SLM

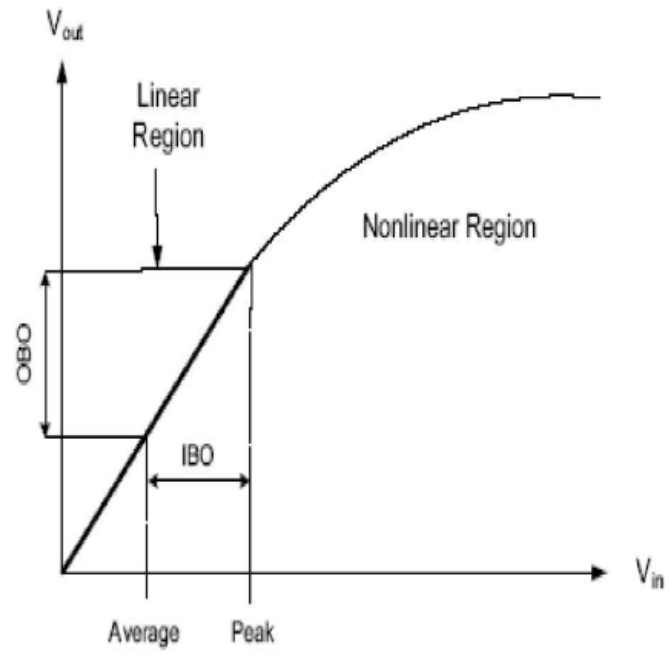

Figure 2:- Linear And Non Linear region

The above figure 2 shows the undesirable nonlinear effects a waveform with a high peak must be transmitted in the linear range of the HPA by decreasing the average power of the input signal. This is called input back-off (IBO) and results in a proportional output back-off (OBO) after the amplification. However high back-offs reduce the power efficiency of the amplifier and cause a reduction in the coverage range. The operating point of the nonlinearity is defined by the input back-off (IBO) that corresponds to the ratio between the saturated and the average input powers. To deal with the high PAPR a number of approaches have been proposed. such as clipping [19] tone reservation , and Selected mapping(SLM) All of the mentioned schemes need an accurate PAPR calculation based on over sampling.

\section{SYSTEM DESCRIPTION}

In an OFDM system, shown [5] data is modulated in the frequency domain to $N$ adjacent sub carriers. These $N$ sub carriers span a bandwidth of $B \mathrm{~Hz}$ and are separated by a spacing of $\Delta f=B / N$.

The continuous-time base band representation of this is

$$
X(n)=\frac{1}{\sqrt{N}} \sum_{k=0}^{N-1} X(k) \frac{e^{j 2 \pi \Delta f k t}}{N}, t=[o, T]
$$

Where $T=1 / f$ is thesymbol period.

\subsection{Peak-to-Average Ratio}

The most popular quantification metric of envelope variation is the peak-to-average ratio (PAR). Rightfully so as PAR captures the most important aspect of a signal that has to pass through a peak-power limited device the peak power. The use of PAR in communications signals is a result of the use of PAR in radar applications. A radar system shares certain similarities with a communications system namely they both have to transmit an amplified radio signal of a certain spectrum. For radar the spectrum shape is often the only signal constraint which makes waveform shaping that minimizes peaks a relatively straightforward problem. However in an OFDM communication system there is the additional constraint that each sub carrier (Fourier coefficient of the spectrum) is modulated with an information bearing complex number. This additional degree of constraint significantly complicates the problem. 


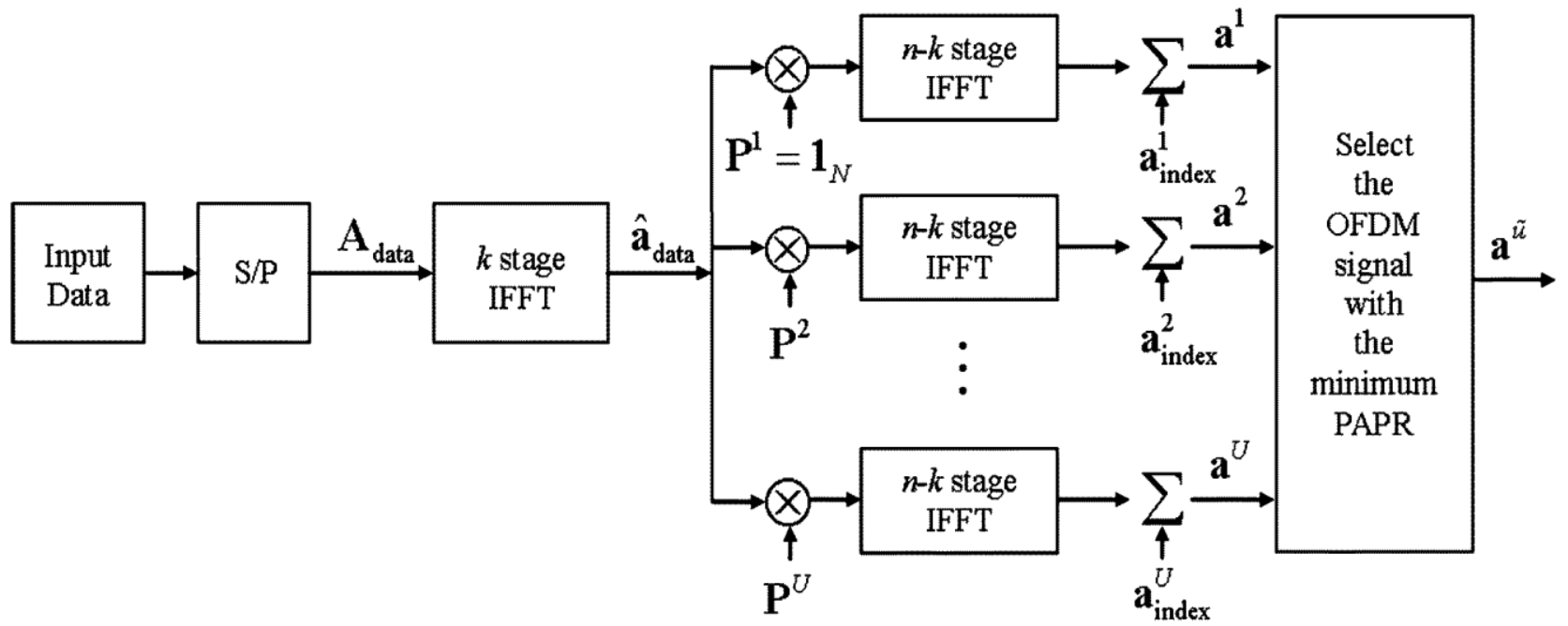

Fig 3.:- New OFDM Scheme

In the discrete time domain, an OFDM signal of carriers can be expressed as

$$
a_{t}=\frac{1}{\sqrt{N}} \sum_{n=0}^{N-1} A_{n} e^{\frac{j 2 \pi n t}{N}}, 0 \leq t \leq N-1
$$

Where $A=\left[A_{o} A_{1} A_{2} \ldots A_{N-1}\right]$ is an input symbol sequence and stands for a discrete time index. The PAPR of an OFDM signal, defined as the ratio of the maximum to the average power of the signal, can be expressed as

$$
\operatorname{PAPR}(a)=\frac{\operatorname{Max}_{(0 \leq t \leq N-1)}\left|a_{t}\right|^{2}}{E\left[\left|a_{t}\right|^{2}\right]}
$$

Define the PAR of an OFDM signal $x$

$$
P A R\{x\}=\frac{M a x|x|^{2}}{E\left[|x|^{2}\right]}
$$

where $x$ and be any signal representation (critically sampled baseband oversampled base-band continuoustime pass band etc.) defined over one symbol period. Because the denominator of an expected value and strictly speaking not an "average" it is true that the term PAR is a bit of a misnomer. Despite this slight technical inaccuracy PAR is the most widely used term and we will keep with convention here. Also note that the ensemble average power and the expectation in the denominator of only differs for non-constant modulus constellations. The PAR of $x[n]$ is different for levels the PAR increases with $N$. First we assume that $N$ the number of subcarriers is large enough so that the discrete-time domain signal has an approximate complex Gaussian distribution [5]. It then follows that the instantaneous power of the discrete- time domain samples is Chi-Squared distributed.

So

$$
\begin{aligned}
\operatorname{Prob}=\left[|X|[n]^{2}\right] & >\gamma] \\
& =1 \\
& -e^{-\sigma^{\wedge} 2 \gamma}
\end{aligned}
$$

the IFFT each discrete time sample can be treated as independent of all other samples. With these two approximations the probability that the power of at least one $x[n]$ out of $N$ samples is above a given level is

$$
\left.\operatorname{Prob}\left[\max |X|[n]^{2}\right]>\gamma\right]=\left(1-e^{-\sigma^{\wedge} 2 \gamma}\right)^{N}
$$

Finally if $E[|x[n]| 2]$ is normalized to unity, then the CCDF of the PAR is

$$
\operatorname{Prob}[\operatorname{PAR}\{X[n]\} \quad>\gamma]=1-\left(1-e^{-\sigma^{\wedge} 2 \gamma}\right)^{N}
$$

We know that OFDM is a promising high-Speed communications technique however it suffers from high PARs.

\section{PAPR REDUCTION}

Ideally a SLM scheme will create D independent mappings of a discrete-time domain signal xL. If we assume that each mapping is independent of all other mappings then the CCDF of the PAR in a SLM scheme is simply

$$
\operatorname{Prob}[\operatorname{PAR}\{X[d]\} \quad>\gamma]=\left[1-\left(1-e^{-\sigma^{\wedge} 2 \gamma}\right)^{N}\right]^{D}
$$

Figure 3 is a plot of the theoretical PAR CCDF curves for a critically sampled OFDM symbol.

where $N=64, \mathrm{~L}=1$ and $\mathrm{d}=1,2,10,100$.

The CCDF of the PAR in a Nyquist sampled OFDM symbol is

$$
\operatorname{Prob}\left[P A R_{S L M}>1\right]=\left[1-\left(1-e^{-\gamma}\right)^{N}\right]^{D}
$$

where $N$ is the number of subcarriers and $D$ is the number of independent phase mappings. 


\subsection{SLM Complexity}

It is obvious that SLM has significant PAR reduction capabilities. However, this reduction is not free. The most significant costs are the D -1 additional IDFT operations and the $D-1, N$ - length phase multiplications. These complexities can be mitigated slightly by using the inverse fast Fourier transform (IFFT) in place of the IDFT and by using binary phase sequences so that all of the phase Multiplications are just sign changed.

\section{THRESHOLD MAPPING}

\section{ALGORITHM}

The goal of PAR reduction is to increase power efficiency while keeping the probability of clipping at an acceptably low level. Clipping needs to be minimized because it is a distorting operation that increases the error rate.

We design an algorithm to achieve the following goals.

We take, our algorithm average power is one in the whole design.

1) Taking a quantifying Clipping according to the clipping $\operatorname{Pr}(P A R>0)$

For future brevity we will define

$$
\begin{aligned}
& U^{N}=\left(1-e^{-\gamma}\right)^{N} \\
& U_{O}^{N}=\left(1-e^{-\gamma_{o}}\right)^{N}
\end{aligned}
$$

2) If $(\mathrm{SLM}==\mathrm{STOP})$

If $\left(P A R<\gamma_{\mathrm{o}}\right)$

Then $\mathrm{D}_{\max }$ confirm the probability to

$$
\begin{aligned}
& \operatorname{Pr}\left(P A R>\gamma_{O}\right) \leq p \\
& p \geq\left(1-U_{O}^{N}\right)^{D_{M A X}} \\
& D_{M A X}=\left[\begin{array}{c}
\ln (p) \\
\ln \left(1-U_{o}^{N}\right)
\end{array}\right]
\end{aligned}
$$

Where $[a]$ is the "ceil" operation that returns the smallest integer greater than or equal to a.

3). If ( $p==t($ Dependent variable $))$ Then the probability is

$$
p=\left(1-U_{O}^{N}\right)^{D_{M A X}}
$$

And if $Y_{o}$ is the dependent variable, then

$$
\gamma_{O}=\ln \left[1-\left(1-e^{\ln \frac{p}{D_{\text {Max }}}}\right)^{\frac{1}{N}}\right]
$$

4) For(;Modernize SLM Scheme)

$$
A^{m}=A_{R}{ }^{m}+j A_{I}^{m}(1 \leq m \leq M)
$$

Where $A_{R}{ }^{m}$ is the real part and $j A_{I}{ }^{m}$ is the imaginary part of the frequency domain vector and $\operatorname{Pr}\left(P A R>\gamma_{\mathrm{o}}\right) \leq P=10^{-.4}$

a SLM system only has to test phase sequences until a signal with a $P A R<\gamma_{o}$ Is found.

5) For (Nyquist sampled OFDM symbol)

$$
\operatorname{Pr}\left[P A R_{S L M}>\gamma\right]=\left[1-\left(1-e^{-\gamma}\right)^{N}\right]^{D}
$$

Where $\mathrm{N}$ is the number of subcarriers and $\mathrm{D}$ is the number of independent phase mapping, for both are generated, since the signals are real valued, the phase vectors $D_{R}$ and $D_{I}$ have to be real.

These candidates are transformed into real domain using IFFTs. Then each combination of one real and one imaginary from these the one candidate with minimum PAR is selected.

6)The CCDF of the PAR in Modernized SLM OFDM Symbol is

$$
\operatorname{Prob}\left[P A R_{S L M}>\gamma\right]_{\text {real }}=\left[1-\left(1-e^{-\gamma}\right)^{N}\right]^{D_{R}}
$$

Where $D_{R}$ is phase sequence for real part

$$
\operatorname{Prob}\left[P A R_{S L M}>\gamma\right]_{\text {imaginary }}=\left[1-\left(1-e^{-\gamma}\right)^{N}\right]^{D_{I}}
$$

Where DI is phase sequence for Imaginary part

$$
\begin{aligned}
\operatorname{Prob}\left[P_{\left.S L R_{S M}>\gamma\right]}\right. & \\
& =\left[1-\left(1-e^{-\gamma}\right)^{N}\right]^{D_{R}} \\
& +\left[1-\left(1-e^{-\gamma}\right)^{N}\right]^{D_{I}}
\end{aligned}
$$

7) The computational complexity reduction ratio (CCRR) of the new SLM OFDM scheme over the conventional SLM OFDM scheme is defined

$$
\begin{aligned}
\text { CCRR } & =\left(1-\frac{\text { Complexity of new SLM }}{\text { Complexity of Conventional SLM }}\right) \times 100 \\
& =\left(1-\frac{1}{U}\right) \frac{k}{n} \times 100
\end{aligned}
$$

In the modernized SLM system we separate the complex baseband signal into real and imaginary part. These real parts converted into serial to parallel and individual phase sequence is multiply into every parallel and selected minimum PAPR. Similarly imaginary parts converted into serial to parallel and individual phase sequence is multiply into every parallel which selects minimum PAPR. Both real and imaginary parts again combine and transmitted.

Finally the computational complexity reduction ratio of the new scheme over the conventional scheme shows a higher computational speed.

\section{CONCLUSION}

In this paper we proposed the modernized SLM, which has a lower PAPR than traditional SLM scheme, via separation of complex baseband signal( frequency domain). We derived the PAR CCDF in a modernized SLM system. Its performance is analyzing in MATLAB 7.8 version. The simulation results show that the MSLM scheme with 1024 carriers and with different phase sequences reduces the PAPR about $0.25 \mathrm{~dB}$ to $1 \mathrm{~dB}$ as compared with conventional SLM Scheme. The computational complexity reduction ratio increase as the phase sequences increases. This makes the proposed scheme more 
suitable for high speed data rate OFDM system.

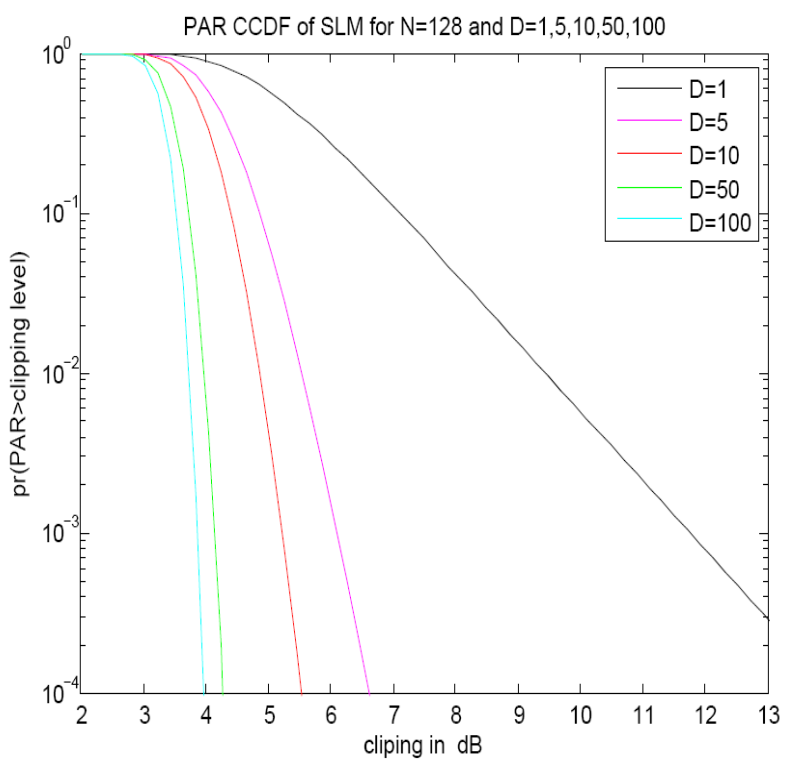

Fig 4:- PAPR CCDF curves for $N=128, L=1$

and $d=1,5,10,50,100$

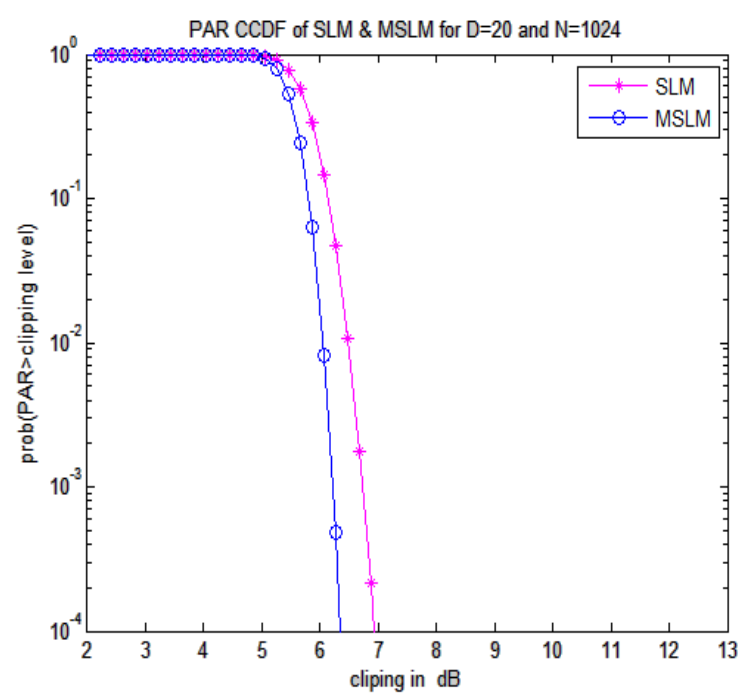

Fig 5:- Simulation results MSLM and SLM

\section{REFERENCE}

[1] M. Breiling, S. H. Müller, and J. B. Huber, "SLM peak power reduction without explicit side information," IEEE Commun. Lett., vol. 5, no. 6,pp. 239-241, Jun. 2001

[2] J. A. Davis and J. Jedwab, "Peak-to-mean power control in OFDM, golay complementary sequences, and ReedMuller codes," IEEE Trans.Inf. Theory, vol. 45, no. 7, pp. 2397- 2417, Nov. 1999

[3] P. V. Eetvelt, G. Wade, and M. Tomlinson, "Peak to average power reducing for OFDM schemes by selective scrambling," Electron Lett., vol. 32, no. 10, pp. 19631964, Oct. 1996
[4] D. W. Lim et al., "On the phase sequences of SLM OFDM system for PAPR reduction," presented at the ISITA, Parma, Italy, Oct. 10-13, 2004,pp. 230-235

[5] S. H. Müller et al., "OFDM with reduced peak-to-average power ratio by multiple signal representation," Ann. Telecommun., vol. 52, no. 1-2, pp. 58-67, Feb. 1997.

[6] J. Tellado and J. Cioffi, PAR reduction in multicarrier transmission systems, in ANSI Document, no. 97-367, pp. 1-14, Dec.8, 1997. T1E1.4 Technical Subcommitte.

[7] Chang $\mathrm{R} \mathrm{W}^{\prime \prime}$ Orthogonal frequency division multiplexing" U.SPatent 3488445 Jan. 1970.

[8] Cripps S C "RF Power Amplifiers for Wireless Communications" Norwood MA Artech House 1999

[9] Breiling M, Muller-Weinfurtner, S. H. and Huber J. B."SLM peak-power reduction without explicit side information" IEEE Communications Letters vol. 5 pp. 239-241 June 2001

[10] Breiling M, Muller-Weinfurtner, S. H. and Huber J. B."SLM peak-power reduction without explicit side information" IEEE Communications Letters vol. 5 pp. 239-241 June 2001

[11] B. S. Krongold and D. L. Jones "PAR reduction in OFDM via active constellation extension" IEEE Trans. Broadcast. vol. 49 no. 3 pp 258-268 Sep. 2002.

[12] Bauml, R.W.; Fischer, R.F.H.; Huber, J.B." Reducing the Peak- to- Average Power Ratio of Multicarrier Modulation by Selected Mapping", Electronic Lett., 32(22):pp.2056-2057, October 1996.

[13] Jayalath, A.D.S.; Tellambura, C.; Wu, H , "Reduced complexity PTS and new phase sequences for SLM to reduce PAP of an OFDM signal", VTC, May 2000, 3, pp. $1914-1917$.

[14] Zhou, G.T.; Baxley, R.J.; Ning Chen; "Selected mapping with monomial phase rotations for peak-to-average power ratio reduction in OFDM", ICCCAS 2004, 1, 27-29 pp:66- 70

[15] Yang Chan Cho; Seung Hee Han; Jae Hong Lee; "Selected mapping technique with novel phase sequences for PAPR reduction of an OFDM signal", VTC Sept. 2004, 7, pp :4781 - 4785.

[16] Dae-Woon Lim et. al. "On the phase sequence set of SLM OFDM scheme for a crest factor reduction”, Signal Processing, IEEE Transactions , 54 (5), May 2006 pp:1931 - 1935 .

[17] Peng Cheng; Yue Xiao; Lilin Dan; Shaoqian Li; "Improved SLM for PAPR Reduction in OFDM System", PIMRC Sept. 2007 pp:1 - 5

[18] Jizeng Wang; Jingyu Luo; Yanlong Zhang; " A New Phase Sequence for SLM in MC-CDMA System", WiCom Sept. 2007 pp:938 - 941.

[19] Mesdagh D and Spruyt P "A method to reduce the probability of clipping in DMT-based transceivers Oct.1996 